\title{
Implications of dietary $\omega-3$ and $\omega-6$ polyunsaturated fatty acids in breast cancer (Review)
}

\author{
OANA ZANOAGA $^{1}$, ANCUTA JURJ ${ }^{1}$, LAJOS RADULY ${ }^{1,2}$, ROXANA COJOCNEANU-PETRIC ${ }^{1}$, \\ ENRIQUE FUENTES-MATTEI ${ }^{3}$, OSCAR WU ${ }^{3,4}$, CORNELIA BRAICU ${ }^{1}$, \\ CLAUDIA DIANA GHERMAN ${ }^{5,6}$ and IOANA BERINDAN-NEAGOE ${ }^{1,7,8}$
}

\begin{abstract}
${ }^{1}$ Research Center for Functional Genomics, Biomedicine and Translational Medicine, Iuliu Hatieganu University of Medicine and Pharmacy, 400337 Cluj-Napoca; ${ }^{2}$ Department of Physiopathology, University of Agricultural Science and Veterinary Medicine, 400372 Cluj-Napoca, Romania; ${ }^{3}$ Department of Experimental Therapeutics, The University of Texas MD Anderson Cancer Center, Houston, TX 77030; ${ }^{4}$ Texas Tech University Honors College, McClellan Hall, Lubbock, TX 79409, USA; ${ }^{5}$ Surgical Clinic II Hospital, 400006 Cluj-Napoca; ${ }^{6}$ Department of Surgery, Iuliu Haţieganu University of Medicine and Pharmacy, 400012 Cluj-Napoca; ${ }^{7}$ MEDFUTURE-Research Center for Advanced Medicine, University of Medicine and Pharmacy Iuliu-Hatieganu, 400349 Cluj-Napoca; ${ }^{8}$ Department of Functional Genomics, Proteomics and Experimental Pathology, Prof Dr Ion Chiricuta Oncology Institute, 400015 Cluj-Napoca, Romania
\end{abstract}

Received May 11, 2017; Accepted July 20, 2017

DOI: $10.3892 /$ etm.2017.5515

\begin{abstract}
Breast cancer represents one of the most common forms of cancer in women worldwide, with an increase in the number of newly diagnosed patients in the last decade. The role of fatty acids, particularly of a diet rich in $\omega-3$ and $\omega-6$ polyunsaturated fatty acids (PUFAs), in breast cancer development is not fully understood and remains controversial due to their complex mechanism of action. However, a large number of animal models and cell culture studies have demonstrated that high levels of $\omega-3$ PUFAs have an inhibitory role in the development and progression of breast cancer, compared to $\omega-6$ PUFAs. The present review focused on recent studies regarding the correlation between dietary PUFAs and breast cancer development, and aimed to emphasize the main molecular mechanisms involved in the modification of cell membrane structure and function, modulation of signal transduction pathways, gene expression regulation, and antiangiogenic and antimetastatic effects. Furthermore, the anticancer role of $\omega-3$ PUFAs through the modulation of microRNA expression levels was also reviewed.
\end{abstract}

Correspondence to: Dr Claudia Diana Gherman, Department of Surgery, Iuliu Haţieganu University of Medicine and Pharmacy, 8 Victor Babes Street, 400012 Cluj-Napoca, Romania

E-mail: gherman.claudia@umfcluj.ro

Key words: breast cancer, $\omega-3$ and $\omega-6$ polyunsaturated fatty acids, signal transduction pathways, microRNA expression, diet and health effects

\section{Contents}

1. Introduction

2. $\omega-3$ and $\omega-6$ fatty acid balance in a healthy diet

3. Implication of $\omega-3$ and $\omega-6$ fatty acids in breast cancer

4. Modifications of cell membrane structure and function

5. Modulation of signal transduction pathways

6. Regulation of gene expression

7. Antimetastatic and antiangiogenic activity

8. Regulation of miRNA expression

9. Conclusion

\section{Introduction}

Breast cancer is a highly prevalent cancer in women worldwide, with $\sim 1.6$ million new cases diagnosed in 2015 (1). Globally, the incidence of breast cancer appears higher in industrialized countries, with the majority of cases being observed in Western Europe, Australia and New Zeeland, and North America (2). According to the National Institute of Statistics, $90 \%$ of women have their disease diagnosed in an advanced form, which dramatically decreases their chances of survival and their quality of life (3-5).

Breast cancer incidence is further increased as a response to multiple toxic environmental exposures or the presence of certain environmental factors, including radiation, mutagens or carcinogens $(6,7)$. Meanwhile, epigenetic and genetic alterations may occur due to an unbalanced diet (1). Mammary cancer development and progression is directly affected by dietary habits and environmental exposure $(1,6,8,9)$. Advances in new generation technologies, particularly in the fields of transcriptomics and metabolomics $(10,11)$, have markedly facilitated the pursuit to elucidate the influence of diet at the molecular level (12). This may eventually contribute to the health evaluation for particular nutritional components, 
with the final purpose of developing novel functional food products $(12,13)$.

Presently there are a broad range of ongoing nutrigenomics studies focusing on detecting the mechanisms on which nutrient and gene interactions are based. Such studies may lead to the identification of genetic variants used for the discovery and development of novel biomarkers for specific and personalized diet prescriptions for each patient $(14,15)$. A classic example is related to the Mediterranean diet, which is associated with reduced mortality rates for a wide range of pathologies, such as cancer (8). According to this, the increased olive oil consumption in a Mediterranean diet is linked to a reduced risk of breast cancer (16-18), due to the beneficial actions of polyunsaturated fatty acids (PUFAs). The main PUFAs are presented in Fig. 1. Therefore, the favorable effects of PUFAs have been demonstrated by epidemiological and experimental studies worldwide. The purpose of the present review was to summarize these findings.

\section{2. $\omega-3$ and $\omega-6$ fatty acid balance in a healthy diet}

It is well known that modern society diets are dominated by processed foods and vegetable oils with high levels of $\omega-6$ and low levels of $\omega-3$ PUFAs (19). A proportion of 2:1 for the case of $\omega-6: \omega-3$ PUFAs is believed to have been present in our ancestors' diet, a ratio that today has been markedly altered to $10: 1$ because of unhealthy dietary habits (14). Overconsumption of $\omega-6$ PUFAs, and an increased proportion of $\omega-6: \omega-3$ PUFA ratio observed in general in Western diets, leads to the activation of pathogenesis mechanisms for a wide range of pathologies (Fig. 2), including cardiovascular diseases, metabolic or immune pathologies, and cancer $(14,20)$. Thus, the risk of cancer may be abridged by limiting the consumption of foods containing $\omega-3$ fatty acids $(21,22)$.

For elongation and desaturation reactions, there is a competition for the same enzymes between the two types of fatty acids. High levels of $\omega-6$ PUFAs result in an inhibition of the elongation and desaturation of $\omega-3$ PUFAs (20-22). This competition between $\omega-3$ and $\omega-6$ PUFAs reveals the importance of low ratios of $\omega-3: \omega-6$, compared to the individual fatty acid concentrations in human organisms (21-29).

\section{Implication of $\omega-3$ and $\omega-6$ fatty acids in breast cancer}

The role of a fatty acid-rich diet in the development and progression of breast cancer is not well understood and remains challenging, particularly since the information from human studies is limited. In vitro cell culture investigations or in vivo animal models have demonstrated the tumor suppressive role of eicosapentaenoic acid (EPA) and docosahexaenoic acid (DHA); however, the inhibitory role of $\omega-3$ PUFAs in cancer is yet to be fully elucidated and requires further investigation $(3,23,24)$.

PUFAs are essential nutrients, and include $\omega-6$ fatty acids, such as linoleic and arachidonic acids (AA), as well as $\omega-3$ fatty acids, including EPA and DHA acids. They have been demonstrated to have notable roles in modulating key cellular and molecular processes (Tables I and II) due to the fact that they are essential precursors of the cell membrane and interfere with other mediators of the inflammatory response $(25,26)$. By adapting the fatty acid composition of the cells, a wide range of aspects related to cell metabolism may be controlled $(24,25)$.

Despite their distinct physiologic and metabolic characteristics, $\omega-6$ and $\omega-3$ PUFAs cannot be endogenously produced by the human body, and thus must be obtained from the diet; however, these should preferably be obtained in the correct ratio (21). This has been supported by multiple epidemiological studies, where a reduced ratio of $\omega-6: \omega-3$ PUFAs has been indicated to have beneficial effects (25-27). Studies have also demonstrated that a modern diet is related to estrogen receptor (ER) negative breast cancer risk among taller women ( $\geq 160 \mathrm{~cm}$ tall) (28).

EPA and DHA are present in marine organisms, particularly in ocean fish. According to a 2011 United Nations report, global fish consumption increased with a yearly average of $17 \mathrm{~kg} /$ person (1,30-32). In the nutritional etiology of breast cancer, $\omega-3$ fatty acids of fish origin have been demonstrated to have a significant role as protective factors, being associated with a $14 \%$ reduction in the risk of developing this malignancy (29-31). When comparing tumor and normal breast tissue in terms of their fatty acid content, higher levels of $\omega-6$ PUFAs were observed in malignant tissues (33). The study conclusion was that an increased expression level of the enzyme $\Delta-6$ desaturase is desired, as well as an abundance of $\omega-6$ PUFA precursors (32).

Preclinical studies have offered a higher understanding of the effects of PUFAs, particularly in the etiology of breast cancer (Fig. 3). These studies have attempted to explain the cancer-related preventive activity of $\omega$-3 PUFAs (Table I), and the association between $\omega-6$ PUFAs and procarcinogenic effects (Table II) in breast malignancies (18), leading to the alteration of gene expression patterns, as well as dysregulations of microRNA (miRNA) sequences. PUFAs were demonstrated to have effects on the composition of the plasma membrane $(18,33,34)$, increased cellular oxidative stress (14), gene expression modifications $(35,36)$, alterations to intracellular signaling pathways $(37,38)$, antiangiogenic and antimetastatic activity (39-43).

\section{Modifications of cell membrane structure and function}

Cell membrane integrity and alterations in signal transduction are important cellular processes in which $\omega-3$ PUFAs are involved, and these cellular changes lead to reduced cell proliferation, the induction of apoptosis and an increased degree of unsaturation (38). Cell membrane structures, their fluidity and permeability, are affected in a notable manner by higher densities of $\omega-6$ fatty acids (44).

High concentrations of $\omega-6$ PUFAs have a potent effect on cell functions by damaging different ion transporters and channels, such as the ones for $\mathrm{Ca}^{2+}(38)$. Increased amounts of w-6 PUFAs reduce the number of $\mathrm{Ca}^{2+}$ channels (45), which damages the fluidity of membranes and affects the function of specific integral and membrane-bound proteins (45-47).

The incorporation of $\omega-3$ PUFAs, particularly EPA and DHA, is able to modify the degree of lipid peroxidation in cell membranes, altering the formation of lipid rafts and suppressing raft-associated cell signal transduction (37). The susceptibility to peroxidation is determined by the degree of unsaturation of the membrane phospholipid fatty acids. High unsaturation causes increased cell oxidative stress and disrupts 


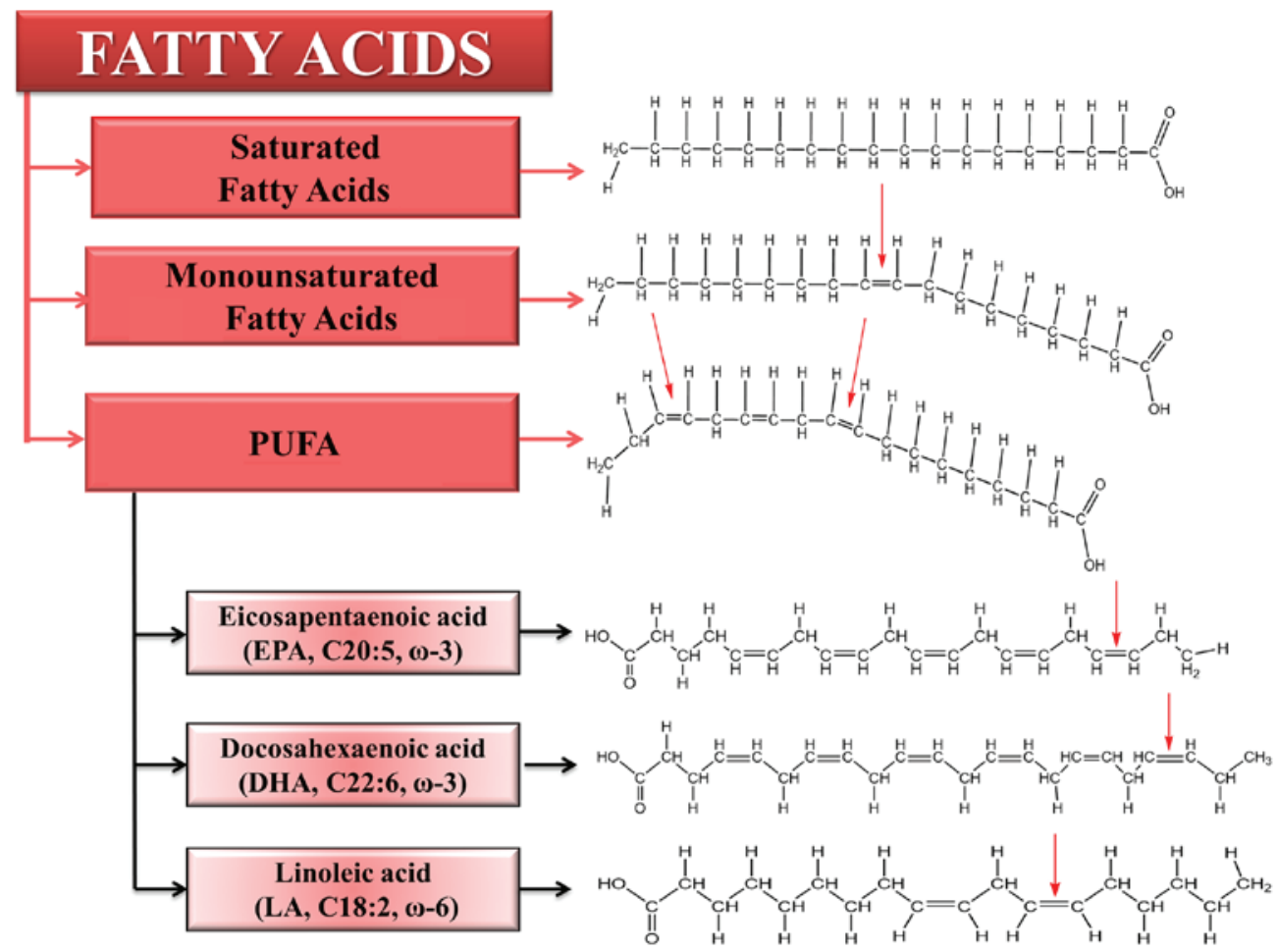

Figure 1. Types of fatty acids with emphasis on the main class of PUFAs. The difference between fatty acids is determined by the presence of double bonds. Eicosapentaenoic acid and docosahexaenoic acid are characterized by the double bond in three positions, also known as $\omega-3$ PUFAs, while linoleic acid has the first double bond in position 6, also known as $\omega-6$ PUFAs. PUFA, polyunsaturated fatty acid.

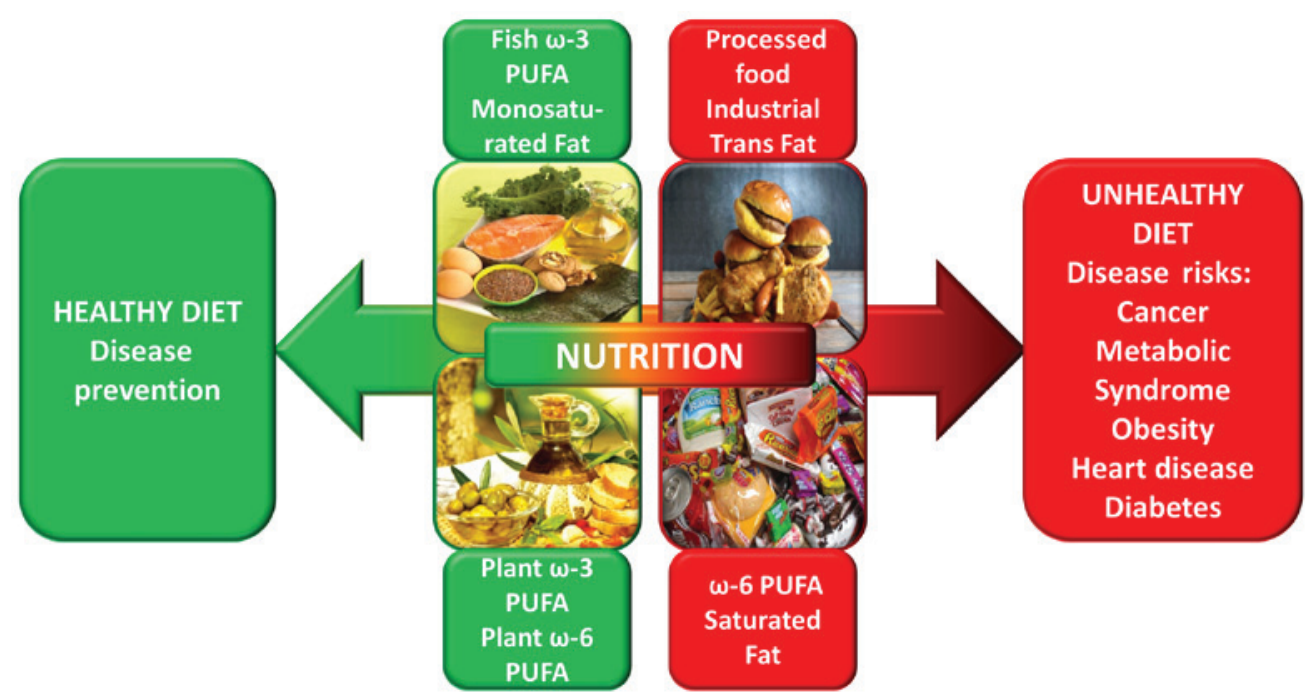

Figure 2. Impact of dietary PUFAs in disease prevention or risk. The diagram emphasizes the importance of a balanced diet to maintain a healthy condition. The human body is unable to synthesize $\omega-6$ and $\omega-3$ PUFAs and they may only be obtained from a balanced diet. The amounts and balance of PUFAs in the diet are important for maintenance of and improving health due to their role in the body's functions, including immune and inflammatory responses, blood lipid levels, blood pressure and blood clotting. PUFA, polyunsaturated fatty acid.

physiological signaling pathways by leading to malignant transformation $(48,49)$.

\section{Modulation of signal transduction pathways}

A clinical study revealed that $\omega-3$ and $\omega-6$ PUFAs have similar biochemical activity and require the same elongase, desaturase, cyclooxygenase and lipoxygenase enzymes (50). Enzymatic conversion into eicosanoids, compounds with notable roles in cell differentiation and growth, is among the most important cellular functions of PUFAs (50-60). Studies have demonstrated that a diet rich in $\omega-6$ PUFAs has strong promoting effects on breast cancer development (50-59). The carcinogenic effects of high levels of $\omega-6$ PUFAs were correlated with increased ratios of eicosanoids (48). Studies have also indicated that eicosanoid compounds, including prostaglandins (PG), thromboxane (TX), leukotrienes (LT), hydroxyl fatty acids and lipoxins, are produced in higher quantities than 
Table I. Principle mechanisms of $\omega-3$ polyunsaturated fatty acids in breast cancer.

\begin{tabular}{llc}
\hline Mechanism & \multicolumn{1}{c}{ Key target/gene } & (Refs.) \\
\hline Changes of cell membrane properties & Bcl-2; procaspase-8 & $(18,37)$ \\
Modulation of intracellular signaling pathways & FAK, NF-kB, MAPK, COX-2 & $(33,82)$ \\
Regulation of gene expression & EGFR, Her-2, Erk 1/2, AKT PTEN, Bcl-2, PDCD4, NF-kB & $(70,110-112)$ \\
Antimetastatic and antiangiogenic activity & EZH2, VEGF, E-cadherin & $(36,103)$ \\
Regulation of miR expression & miR-21, miR-26a/b, miR19b, miR146b, miR183 & $(34,42,110)$
\end{tabular}

Bcl-2, B-cell lymphoma 2; FAK, focal adhesion kinase; NF- $\kappa$ B, nuclear factor $\kappa \mathrm{B}$; MAPK, mitogen-activated protein kinase; COX-2, cyclooxygenase 2; EGFR, epidermal growth factor receptor; Erk, extracellular signal-regulated kinase; PTEN, phosphatase and tensin homolog; PDCD4, programmed cell death 4; EZH2, enhancer of zeste 2; VEGF, vascular epithelial growth factor; miR, microRNA.

Table II. Principle mechanisms related to pro-carcinogenic effects of $\omega-6$ polyunsaturated fatty acids in breast cancer.

\begin{tabular}{llc}
\hline Mechanism & \multicolumn{1}{c}{ Key/target gene } & (Refs.) \\
\hline Lipid peroxidation, DNA adducts & Redox-cycling of 4-hydroxyestradiol & $(21,26,37)$ \\
Regulation of gene expression & p21WAF1/CIP1, MAPK, TGF- $\beta$, TLR & $(21,42)$ \\
Antimetastatic and antiangiogenic activity & VEGF, FGF, HIF- $\alpha$, E-cadherin & $(21,41,122)$ \\
Regulation of miR expression & MiR19b, miR146b, miR1835p, let-7a, & $(42,109)$ \\
& miR-23b, miR-27a/b, miR-21, let-7 &
\end{tabular}

MAPK, mitogen-activated protein kinase; TGF- $\beta$, transforming growth factor- $\beta$; TLR, toll-like receptor; VEGF, vascular epithelial growth factor; FGF, fibroblast growth factor; HIF- $\alpha$, hypoxia-inducible factor- $\alpha$; miR, microRNA.

those of $\omega$-3 PUFAs, due to the high amounts of $\omega-6$ PUFAs present in Western diets $(20,51,52)$.

Eicosanoids derived from $\omega-6$ PUFAs have been demonstrated to have pro-inflammatory and pro-carcinogenic effects as compared to $\omega-3$ PUFA-derived lipid mediators $(48,53)$. In obese individuals, $\omega-6$ PUFA-derived eicosanoid levels were observed to be increased, which stimulated breast cancer initiation, invasion and metastasis (54).

Cyclooxygenase (COX)-2 is an enzyme that serves an active role in prostaglandin synthesis, and increased levels are associated with inflammation in all subtypes of breast cancer (55-57). The COX and lipoxygenase (LOX) enzymes are key factors for the enzymatic production of PG and LT (58). $\omega-3$ fatty acids compete with $\omega-6$ fatty acids for COXs for the production of eicosanoids, and a suppressive effect on COX-2 expression has been observed (59-62). COX enzymes produce two-series prostanoids, including PG and TX, and four-series LT, including LTC4, LTD4, LTE4 and LTF4, while LOX enzymes produce hydroxyeicosatetraenoic acids $(48,63)$. In a transgenic mouse model expressing human epidermal growth factor receptor 2 (HER2)/neu, treatment with dietary $\omega-3$ PUFAs inhibited breast tumor cell proliferation and upregulated COX-2 expression (64).

In breast tissue, the metabolites of the arachidonate 5-lipoxygenase pathway are able to induce tumorigenesis and sustain breast cancer progression (65-68). A study on LOX genetic variants combined with $\omega$-6PUFAs revealed a significant increase of breast cancer risk (14). According to a study on the breast cancer cell line MCF-7, DHA upregulated syndecan-1 (a component of the extracellular matrix) expression and promoted apoptosis via downregulation of MEK/extracellular signal-regulated kinases (Erk)/Bad signaling (69). Another study indicated that $\omega-3$ PUFA treatment reduced the effect of E2 on epidermal growth factor receptor (EGFR), Erk1/2 and AKT, and upregulated $\mathrm{G}$ protein-coupled estrogen receptor 1 (GPER1)-cyclic adenosine 5'-phosphate (cAMP)-protein kinase A (PKA) signaling (70). In MDA-MB-231 breast cancer cells, linoleic acid (LA) induces focal adhesion kinase (FAK) activation and cell migration by modulating a FAK-dependent pathway (33).

\section{Regulation of gene expression}

Evidence-based preclinical studies and epidemiologic data consistently support the anticancer effect of $\omega-3$ PUFAs based on their capacity to target key genes altered in breast cancer (71-78). EGFR and HER2 are cell surface receptor tyrosine kinases, representing key therapeutic targets in breast cancer management $(39,79,80)$. $\omega-3$ PUFAs may represent a dietary approach for controlling growth factor-mediated carcinogenesis, by activating tyrosine kinase transduction pathways, p38 mitogen-activated protein kinase activation and apoptosis induction (71). Restoring EGFR signaling was observed in many breast cancer cases as being correlated with dietary habits (72), while an apoptotic effect of DHA from marine sources was found by targeting EGFR pathways in malignant breast tissue (38). In MCF-7 and T47D cells, $\omega-3$ PUFA treatment may initiate pro-apoptotic signaling of estrogen by increasing the GPER1-cAMP-PKA signaling response, and inhibiting EGFR, Erk1/2 and AKT activity (70). 
Overexpression of the tyrosine kinase receptor, ErbB2/HER2/neu, occurs in 25-30\% of invasive breast cancer cases with poor prognosis (73). HER2/neu is an oncogene that is overexpressed in many types of cancer, with an important role in development, progression and chemosensitivity of tumors; studies have demonstrated that it is downregulated by $\omega-3$ PUFAs $(73,74)$.

As described in previous studies, $\omega-3$ PUFA effects are also observed at the translational and post-translational level. In mammary cancer cell lines (MCF10A, MCF7, T47D and MDA-MB-231), $\omega-3$ PUFAs may modulate the protein expression of the transcription regulator enhancer of zeste 2 polycomb repressive complex 2 subunit (36), while the activation of peroxisome proliferator-activated receptors (PPAR) was induced in the same type of cancer cells (75). PPARs (PPAR $\alpha$, PPAR $\gamma$ and PPAR $\beta / \delta$ ) are ligand-activated transcription factors of the nuclear hormone receptor superfamily involved in glucose and fatty acid metabolism (76). This PUFA-mediated PPAR activation exerts an effect on several molecular mechanisms, including apoptosis and autophagy $(36,81-84)$. PPAR $\beta$ expression was reduced by a $\omega-3$ PUFA-rich diet in mammary tumors, while in other circumstances, the expression of other PPAR mRNA was modulated, leading to the inhibition of breast cancer cell growth (35). In MCF-7 breast cancer cells, $\omega$-3 PUFA ethanolamides, docosahexaenoyl ethanolamine (DHEA) and eicosapentaenoyl ethanolamine (EPEA), augmented the expression of PPAR $\gamma$, inducing autophagy (77). At the same time, in MCF-7 and MDA-MB-231 cells, AA decreased the Erk1/2 phosphorylation level, and positively modulated PPAR $\gamma$ and PPAR $\alpha$ expression (78). In MCF-7 cells, DHEA and EPEA stimulated the expression of PPAR $\gamma$ as well as PPAR response element-dependent transcription by upregulating phosphatase and tensin homolog (PTEN) expression, while inhibiting the AKT-mechanistic target of the syntetic agent rapamycin via mTOR pathway (77). In another study, MCF-7 cells treated with DHA from a cultured microalga demonstrated increased apoptosis via the upregulation of the B-cell lymphoma 2 (Bcl-2)-associated X protein/Bcl-2 ratio, and inhibition of cell growth (79). In a rat model of breast cancer, dietary $\omega-3$ PUFAs increased the apoptotic index in tumor cells $(80,81)$.

AA induces nuclear factor (NF) $\mathrm{BB}-\mathrm{DNA}$ binding activity through a phosphoinositide 3-kinase- and AKT-dependent pathway (82). $\omega-3$ PUFAs were demonstrated to modulate total AKT expression (83). In contrast with $\omega$-6 PUFAs, $\omega$-3 PUFAs reduced $\mathrm{COX}-2$ and $\mathrm{NF \kappa B}$ expression, decreasing the level of cell invasiveness (84). LA induces FAK and NFKB activation, migration and invasion in MCF10A human mammary epithelial cells (85).

Another fundamental process related to carcinogenesis is cell proliferation. $\mathrm{Ki}-67$ is a nuclear protein used as a prognostic or predictive marker in breast cancer (86). Treatment with $\alpha$-linolenic acid (ALA)-rich flaxseed oil in rats induced a decrease in tumor size, together with decreased Ki-67 levels (87,88). Proliferating cell nuclear antigen (PCNA) is also considered by researchers to be a potential prognostic marker in breast cancer (89). It has been demonstrated that diets rich in $\omega-3$ PUFAs reduce the percentage of proliferating tumor cells by decreasing the expression levels of PCNA (90).

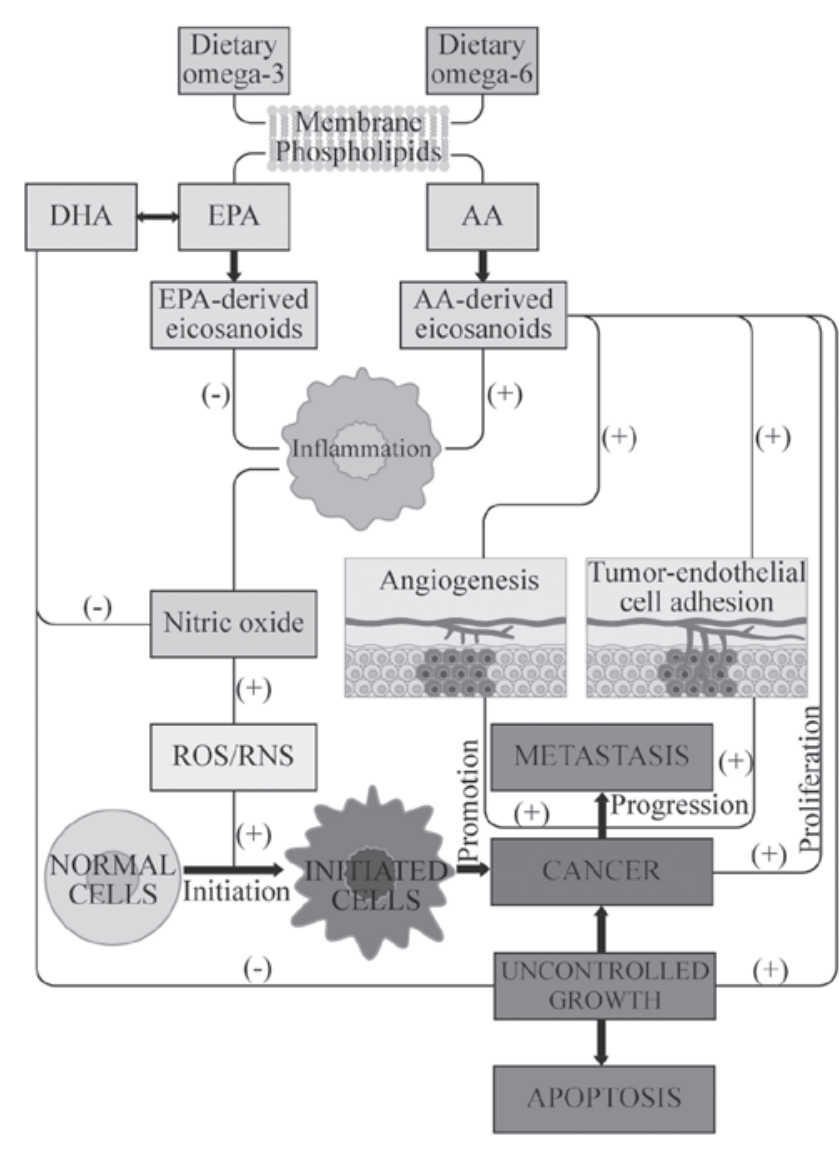

Figure 3. Potential mechanisms of action of $\omega-3$ and $\omega-6$ PUFAs in tumorigenesis, related to the activation of inflammation and production of ROS, which finally leads to the activation of cell proliferation, a predisposition for carcinogenesis and distant metastasis in breast cancer. PUFAs may stimulate (+) or suppress (-) pathways. Dietary $\omega-3$ PUFAs suppress the inflammatory process, stimulate apoptosis, inhibit metastasis and tumor proliferation, and also upregulate the gene expression of antioxidant enzymes. In tumor cells, phospolipase A2, cyclooxygenase 2 and lipoxygenases are overexpressed and induce the overproduction of AA (20:4n-6)-derived eicosanoids, which lead to inflammatory processes. The production of nitric oxide is elevated in inflammation and is involved in the initiation and the progression of carcinogenesis. Nitric oxide may be responsible for tumor growth and metastasis due to its ability to stimulate tumor cell angiogenesis. $\omega$-3 PUFAs reduce the desaturation and elongation of linoleic acid (18:2n-6) to AA. ROS, reactive oxygen species; PUFA, polyunsaturated fatty acid; AA, arachidonic acid; EPA, eicosapentaenoic acid; DHA, docosahexaenoic acid; RNS, reactive nitrogen species.

Studies have indicated that PUFAs have an effect on lipid metabolism in mammary tumors by modifying the expression levels of fatty acid binding protein 5, cluster of differentiation 36, FAS and ER genes (91). The alterations of gene expression levels were demonstrated to be time-dependent in MDA-MB-231 cells following ALA treatment, accompanied by low levels of ID1, and increased JUN, NME1 and thrombospondin 1 expression (92). A significant increase of Erk1/2 and AKT phosphorylation levels was observed in MCF-7 cells treated with a combination of tamoxifen and $\omega-3$ PUFA, compared to $\omega$-3 PUFA alone (93). Additionally, mammary tumor growth and Py 230 cancer cell proliferation was inhibited by $\omega-3$ PUFAs, independent of GPR 120 signaling, suggesting that $\omega-3$ PUFAs act in a Toll-like receptor 4-mediated fashion, or via peroxisome proliferator-activated receptors or other G protein-coupled receptors (94). 


\section{Antimetastatic and antiangiogenic activity}

Different fatty acid compositions affect the affect the breast cancer carcinogenic mechanisms, in particular those associated with tumor growth, proangiogenic and metastatic capacities in breast cancer cells $(95,96)$. In HT115 and MDA-MB-231, $\omega-6$ PUFAs enhanced the expression of a metastasis-suppressor gene, nm-23 (97). Studies conducted on various animal models have demonstrated the effect of PUFAs in regards to cellular growth. A reduction in tumor growth and proliferative abilities caused by PUFAs was observed in an immunocompromised nude murine model of transplanted human breast cancer cells (98). A fish oil diet rich in EPA and DHA in a murine model of MDA-MB-231 human breast cancer cells led to the prevention of bone metastases (99).

The molecular mechanism by which the administration of PUFAs, alone or alongside other compounds, may affect the metastatic potential of tumors remains to be deciphered. The anti-proliferative and anti-invasion activity of DHA may be connected to alterations in the composition of fatty acids, which leads to damage to the membranes of tumor cells, and consequently reduction in metastatic potential $(96,100,101)$.

A diet rich in fatty acids (DHA and EPA) of marine origin in patients with breast cancer was correlated with reduced mortality (102). $\omega-3$ PUFAs lead to E-cadherin expression upregulation, while inhibiting the invasion mechanisms in mammary malignant cells (36), since the appropriate expression of E-cadherin is important in maintaining the integrity of intracellular adhesions (103).

In human breast tumor tissues, AA and cytosolic phospholipase A2 were demonstrated to be associated with the signaling activity of mTOR complex (C) 1 and mTORC 2 , and with expression levels of vascular epithelial growth factor (68).

\section{Regulation of miRNA expression}

The mechanisms by which dietary factors modulate the expression of miRNA in breast cancer cells have not been completely elucidated (104-106). Experimental studies have suggested that some nutrients, including $\omega-3$ PUFAs (107-109), have anticancer effects through the modulation of miRNA expression levels (110-113). Previous studies have demonstrated extensive interactions between $\omega-3$ PUFAs and miRNA in cancer, lipid metabolism and inflammation (105). Recent findings indicated that DHA, which has anti-inflammatory and anticancer effects, is able to downregulate miRNA-21, causing an increase in tumor necrosis factor $\alpha$ mRNA expression levels and, subsequently, triggering apoptosis in human cancer cells $(106,107)$. High expression of miRNA-21 is strongly correlated with poor prognosis in breast cancer, demonstrating a negative impact on overall survival and disease/recurrence-free survival $(104,106,108)$. As part of its mechanism of action, DHA treatment promotes inhibition of receptor-interacting protein 1 kinase and AMP-activated protein kinase- $\alpha$, resulting in nuclear accumulation of Foxo3a, which, in turn, binds to the miRNA-21 promoter causing its transcriptional repression (107). Other studies have demonstrated that expression levels of certain miRNA, including let-7a, miRNA-23b, miRNA-27a/b, miRNA-21, let-7 and miRNA-320b, in breast cancer cell exosomes have been increased by DHA treatment (47-109). An in vitro study on mammary cancer cell models indicated that DHA treatment inhibited the expression of colony stimulating factor 1 and miRNA-21, supporting the evidence found by an in vivo study (110). In MCF-7 and MDA-MB-231 breast cancer cell lines, the promoter of miRNA-21 contains a NFkB binding element, which, in association with the DHA treatment, leads to decreased miRNA-21 expression levels by inhibiting NFKB activity $(105,110)$. Although the mechanisms by which $\omega-3$ PUFAs contribute to the altered expression of these miRNA remain unclear, some authors suggest that its direct targets are involved, together with other associated proteins, including PTEN, Bcl-2, programmed cell death 4 and NFKB (110-112).

A PUFA-enriched diet correlates with changes in circulating miRNA (upregulated miRNA include miRNA-18a, -19b, -106a, -130b, -192, -486-5p and -769-5p; downregulated miRNA include miRNA-125a-5p, $-221,-328$ and $-330-3 p$ ) that may serve an important role in the PUFA dietary systemic effect (113). In a rat model of inflammation, dietary $\omega-3$ and ( -6 PUFAs may alter the miRNA expression profile (42). Functional analyses of these changes in miRNA expression profiles have indicated that dietary PUFAs are implicated in the maintenance of immune homeostasis via the expression of miRNA (43). In same study by Zheng et al (42), it was demonstrated that $\omega-3$ PUFAs suppress inflammation in vivo by inhibiting the expression levels of miR-18-5p, -19b-3p and $-146 b-5 p$.

In addition to inflammation homeostasis, $\omega-3$ PUFAs have also been implicated in the downregulation of miRNA-26a/b expression, promoting the upregulation of 15-hydroxyprostaglandin dehydrogenase, which catalyzes the oxidation of the pro-inflammatory lipid mediator, prostaglandin E2, leading to a decrease in cell proliferation (34). DHA may positively modulate expression levels of miRNA related to lipid metabolism, including miRNA-30c and -192, in cancer and obesity (114-117). Studies in breast cancer have demonstrated that miRNA-30c negatively regulates NFKB signaling and cell cycle progression (118), while miRNA-192 inhibits cell proliferation (119). Knockdown of DICER in enterocyte Caco-2 cells exposed to DHA lipid micelles revealed multiple genes regulating lipid metabolism that are modulated by miRNA-30c and miR-192 (117). miR-33a and miR-122 expression levels in the liver are upregulated in rats with cafeteria-diet induced dyslipidemia; however, these levels are counteracted by the presence of $\omega-3$ PUFAs in vivo (120).

The beneficial effects of $\omega-3$ PUFAs extend to its related metabolites, such as Resolvin D1 (RvD1), which is a well-known anti-inflammatory agent that induces upregulation of miRNA-208a and miR-219 in in vivo transgenic mice overexpressing $\mathrm{N}$-formyl peptide receptor 2 (121). A study by Krishnamoorthy et al (121) indicated that this RvD1-induced upregulation of miRNA-208a promotes increased secretion levels of the anti-inflammatory cytokine, interleukin-10, in human macrophages.

\section{Conclusion}

Dietary factors, such as fatty acids, have been recognized as influential factors in the activation of carcinogenic events or disease progression, and have been associated with a direct 
connection to breast cancer prevention. PUFAs differentially inhibit mammary tumor development by inflicting modifications to the morphology of cell membranes, and influencing signaling pathways, gene expression and apoptosis. Observing the molecular mechanisms involved in the activity of dietary PUFAs on breast cancer development and progression suggests that dietary supplements, in combination with anticancer drugs, should be provided under medical supervision. The majority of studies recommend that patients consume a diet rich in $\omega-3$ PUFAs, while reducing the intake of $\omega-6$ PUFAs, particularly in the case of chemoprevention purposes. Therefore, modification of dietary habits, particularly regarding the choice and amounts of fats consumed, may be used as a strategy for breast cancer prevention. For better results in this field, additional clinical trials are required to evaluate the specific effects of PUFAs on breast cancer outcomes.

\section{Acknowledgements}

The present study was part of the research 'New strategies for improving life quality and survival in cancer patients: Molecular and clinical studies of the tumor genome in deuterium-depleted water treatment augmentation-GenCanD' (grant no. 128/2014; PN-II-PT-PCCA-2013-4-2166) and 'Targeting the TGF $\beta$ pathway: An alternative for breast cancer therapy; POSCCE 709 BreastImpact' (grant no. PN-II-RU-TE-2014-4-1464, 307 from 01/10/2015).

\section{References}

1. Fenga C: Occupational exposure and risk of breast cancer Biomed Rep 4: 282-292, 2016.

2. Irimie A, Achimas-Cadariu P, Burz C and Puscas E: Multiple primary malignancies-epidemiological analysis at a single tertiary institution. J Gastrointestin Liver Dis 19: 69-73, 2010.

3. Bougnoux P, Hajjaji N, Maheo K, Couet C and Chevalier S: Fatty acids and breast cancer: Sensitization to treatments and prevention of metastatic re-growth. Prog Lipid Res 49: 76-86, 2010.

4. Tourassi G, Yoon HJ and Xu S: A novel web informatics approach for automated surveillance of cancer mortality trends. J Biomed Inform 61: 110-118, 2016.

5. McKay D, Kim SK, Taylor S, Abramowitz JS, Tolin D, Coles M, Timpano KR and Olatunji B: An examination of obsessive-compulsive symptoms and dimensions using profile analysis via multidimensional scaling (PAMS). J Anxiety Disord 28 : 352-357, 2014.

6. Snedeker SM: Chemical exposures in the workplace: Effect on breast cancer risk among women. AAOHN J 54: 270-279; quiz 280-281, 2006

7. Braicu C, Berindan-Neagoe I, Pileczki V, Cojocneanu-Petric R, Pop LA, Puscas E, Irimie A and Buiga R: Breast tumor bank: An important resource for developing translational cancer research in Romania. Cancer Biomark 14: 119-127, 2014.

8. Carruba G, Cocciadiferro L, Di Cristina A, Granata OM, Dolcemascolo C, Campisi I, Zarcone M, Cinquegrani $\mathrm{M}$ and Traina A: Nutrition, aging and cancer: Lessons from dietary intervention studies. Immun Ageing 13: 13, 2016.

9. Braicu C, Chiorean R, Irimie A, Chira S, Tomuleasa C, Neagoe E, Paradiso A, Achimas-Cadariu P, Lazar V and Berindan-Neagoe I: Novel insight into triple-negative breast cancers, the emerging role of angiogenesis, and antiangiogenic therapy. Expert Rev Mol Med 18: e18, 2016.

10. Irimie AI, Braicu C, Cojocneanu-Petric R, Berindan-Neagoe I and Campian RS: Novel technologies for oral squamous carcinoma biomarkers in diagnostics and prognostics. Acta Odontol Scand 73: 161-168, 2015.

11. Braicu C, Cojocneanu-Petric R, Chira S, Truta A, Floares A, Petrut B, Achimas-Cadariu P and Berindan-Neagoe I: Clinical and pathological implications of miRNA in bladder cancer. Int J Nanomedicine 10: 791-800, 2015.
12. Fitó $\mathbf{M}$ and Konstantinidou V: Nutritional genomics and the mediterranean diet's effects on human cardiovascular health. Nutrients 8: 218, 2016.

13. Ordovas JM, Kaput J and Corella D: Nutrition in the genomics era: Cardiovascular disease risk and the Mediterranean diet. Mol Nutr Food Res 51: 1293-1299, 2007.

14. Simopoulos AP: Genetic variants in the metabolism of omega-6 and omega-3 fatty acids: Their role in the determination of nutritional requirements and chronic disease risk. Exp Biol Med (Maywood) 235: 785-795, 2010.

15. Minich DM and Bland JS: Personalized Lifestyle Medicine: Relevance for nutrition and lifestyle recommendations. ScientificWorldJournal 2013: 129841, 2013.

16. High olive oil consumption linked to lower breast cancer risk Harv Womens Health Watch 23: 8, 2015.

17. Filik L and Ozyilkan O: Olive-oil consumption and cancer risk. Eur J Clin Nutr 57: 191, 2003.

18. Corsetto PA, Montorfano G, Zava S, Jovenitti IE, Cremona A, Berra B and Rizzo AM: Effects of n-3 PUFAs on breast cancer cells through their incorporation in plasma membrane. Lipids Health Dis 10: 73, 2011.

19. Grosso G, Pajak A, Marventano S, Castellano S, Galvano F, Bucolo C, Drago F and Caraci F: Role of Omega-3 fatty acids in the treatment of depressive disorders: A comprehensive meta-analysis of randomized clinical trials. PLoS One 9: e96905, 2014.

20. Simopoulos AP: The importance of the omega-6/omega-3 fatty acid ratio in cardiovascular disease and other chronic diseases. Exp Biol Med (Maywood) 233: 674-688, 2008.

21. Mansara PP, Deshpande RA, Vaidya MM and Kaul-Ghanekar R: Differential ratios of omega fatty acids (AA/EPA+DHA) modulate growth, lipid peroxidation and expression of tumor regulatory MARBPs in breast cancer cell lines MCF7 and MDA-MB-231. PLoS One 10: e0136542, 2015.

22. Negi AK, Bhatnagar A and Agnihotri N: Fish oil augments celecoxib mediated alteration in apoptotic pathway in the initiation phase of 7,12-dimethylbenz $(\alpha)$ anthracene-induced mammary carcinogenesis. Biomed Pharmacother 79: 9-16, 2016.

23. Serini S, Fasano E, Piccioni E, Cittadini AR and Calviello G: Differential anti-cancer effects of purified EPA and DHA and possible mechanisms involved. Curr Med Chem 18: 4065-4075, 2011.

24. Merendino N, Costantini L, Manzi L, Molinari R, D'Eliseo D and Velotti F: Dietary $\omega-3$ polyunsaturated fatty acid DHA: A potential adjuvant in the treatment of cancer. Biomed Res Int 2013: 310186, 2013.

25. Simopoulos AP: An Increase in the Omega-6/Omega-3 fatty acid ratio increases the risk for obesity. Nutrients 8: 128, 2016.

26. Sun X, Nair J, Linseisen J, Owen RW and Bartsch H: Lipid peroxidation and DNA adduct formation in lymphocytes of premenopausal women: Role of estrogen metabolites and fatty acid intake. Int J Cancer 131: 1983-1990, 2012.

27. Bassett JK, Hodge AM, English DR, MacInnis RJ and Giles GG: Plasma phospholipids fatty acids, dietary fatty acids, and breast cancer risk. Cancer Causes Control 27: 759-773, 2016.

28. Hidaka BH, Kimler BF, Fabian CJ and Carlson SE: An empirically derived dietary pattern associated with breast cancer risk is validated in a nested case-control cohort from a randomized primary prevention trial. Clin Nutr ESPEN 17: 8-17, 2017

29. Zheng JS, Hu XJ, Zhao YM, Yang J and Li D: Intake of fish and marine n-3 polyunsaturated fatty acids and risk of breast cancer: Meta-analysis of data from 21 independent prospective cohort studies. BMJ 346: f3706, 2013.

30. Kim J, Lim SY, Shin A, Sung MK, Ro J, Kang HS, Lee KS, Kim SW and Lee ES: Fatty fish and fish omega-3 fatty acid intakes decrease the breast cancer risk: A case-control study. BMC cancer 9: 216, 2009.

31. Iyengar NM, Hudis CA and Gucalp A: Omega-3 fatty acids for prevention of breast cancer: An update and the state of the science. Curr Breast Cancer Rep 5: 247-254, 2013.

32. Pender-Cudlip MC, Krag KJ, Martini D, Yu J, Guidi A, Skinner SS, Zhang Y, Qu X, He C, Xu Y, et al: Delta-6-desaturase activity and arachidonic acid synthesis are increased in human breast cancer tissue. Cancer Sci 104: 760-764, 2013.

33. Serna-Marquez N, Villegas-Comonfort S, Galindo-Hernandez O, Navarro-Tito N, Millan A and Salazar EP: Role of LOXs and COX-2 on FAK activation and cell migration induced by linoleic acid in MDA-MB-231 breast cancer cells. Cell Oncol (Dordr) 36: 65-77, 2013. 
34. Yao L, Han C, Song K, Zhang J, Lim K and Wu T: Omega-3 polyunsaturated fatty acids upregulate $15-\mathrm{PGDH}$ expression in cholangiocarcinoma cells by inhibiting $\mathrm{miR}-26 \mathrm{a} / \mathrm{b}$ expression. Cancer Res 75: 1388-1398, 2015.

35. Wannous R, Bon E, Mahéo K, Goupille C, Chamouton J, Bougnoux P, Roger S, Besson P and Chevalier S: PPAR $\beta$ mRNA expression, reduced by n-3 PUFA diet in mammary tumor, controls breast cancer cell growth. Biochim Biophys Acta 1831: $1618-1625,2013$

36. Dimri M, Bommi PV, Sahasrabuddhe AA, Khandekar JD and Dimri GP: Dietary omega-3 polyunsaturated fatty acids suppress expression of EZH2 in breast cancer cells. Carcinogenesis 31 489-495, 2010

37. Rogers KR, Kikawa KD, Mouradian M, Hernandez K, McKinnon KM, Ahwah SM and Pardini RS: Docosahexaenoic acid alters epidermal growth factor receptor-related signaling by disrupting its lipid raft association. Carcinogenesis 31 : $1523-1530,2010$

38. Corsetto PA, Montorfano G, Zava S, Jovenitti IE, Cremona A, Berra B and Rizzo AM: Effects of n-3 PUFAs on breast cancer cells through their incorporation in plasma membrane. Lipids Health Dis 10: 73, 2011

39. Merendino N, Costantini L, Manzi L, Molinari R, D'Eliseo D and Velotti F: Dietary $\omega-3$ polyunsaturated fatty acid DHA A potential adjuvant in the treatment of cancer. Biomed Res Int 2013: 310186, 2013.

40. Xu Y and Qian SY: Anti-cancer activities of $\omega-6$ polyunsaturated fatty acids. Biomedical J 37: 112-119, 2014.

41. Rose DP and Connolly JM: Regulation of tumor angiogenesis by dietary fatty acids and eicosanoids. Nutr Cancer 37: 119-127, 2000.

42. Zheng Z, Ge Y, Zhang J, Xue M, Li Q, Lin D and Ma W: PUFA diets alter the microRNA expression profiles in an inflammation rat model. Mol Med Rep 11: 4149-4157, 2015.

43. Onisim A, Achimas-Cadariu A, Vlad C, Kubelac P and Achimas-Cadariu P: Current insights into the association of Nestin with tumor angiogenesis. J BUON 20: 699-706, 2015.

44. Wassall SR and Stillwell W: Docosahexaenoic acid domains: The ultimate non-raft membrane domain. Chem Phys Lipids 153 57-63, 2008

45. Belevych AE, Ho HT, Terentyeva R, Bonilla IM, Terentyev D, Carnes CA, Gyorke S and Billman GE: Dietary omega-3 fatty acids promote arrhythmogenic remodeling of cellular $\mathrm{Ca} 2+$ handling in a postinfarction model of sudden cardiac death. PLoS One 8: e78414, 2013.

46. Stillwell W, Shaikh SR, Zerouga M, Siddiqui R and Wassall SR Docosahexaenoic acid affects cell signaling by altering lipid rafts. Reprod Nutr Dev 45: 559-579, 2005.

47. Nielsen G, Wandall-Frostholm C, Sadda V, Oliván-Viguera A, Lloyd EE, Bryan RM Jr, Simonsen U and Köhler R: Alterations of N-3 polyunsaturated fatty acid-activated K2P channels in hypoxia-induced pulmonary hypertension. Basic Clin Pharmacol Toxicol 113: 250-258, 2013.

48. Larsson SC, Kumlin M, Ingelman-Sundberg $M$ and Wolk A Dietary long-chain n-3 fatty acids for the prevention of cancer: A review of potential mechanisms. Am J Clin Nutr 79: 935-945, 2004.

49. Owen RW, Haubner R, Wurtele G, Hull E, Spiegelhalder B and Bartsch H: Olives and olive oil in cancer prevention. Eur J Cancer Prev 13: 319-326, 2004

50. Fabian CJ and Kimler BF: Marine-derived omega-3 fatty acids: Fishing for clues for cancer prevention. Am Soc Clin Oncol Educ Book: 97-101, 2013.

51. Simopoulos AP: The importance of the ratio of omega-6/omega-3 essential fatty acids. Biomed Pharmacother 56: 365-379, 2002

52. Wysoczański T, Sokoła-Wysoczańska E, Pekala J, Lochyński S, Czyż K, Bodkowski R, Herbinger G, Patkowska-Sokoła B and Librowski T: Omega-3 fatty acids and their role in central nervous system-a review. Curr Med Chem 23: 816-831, 2016.

53. Calder PC: The role of marine omega-3 (n-3) fatty acids in inflammatory processes, atherosclerosis and plaque stability. Mol Nutr Food Res 56: 1073-1080, 2012.

54. Vona-Davis L and Rose DP: The obesity-inflammation-eicosanoid axis in breast cancer. J Mammary Gland Biol Neoplasia 18: 291-307, 2013.

55. Miglietta A, Toselli M, Ravarino N, Vencia W, Chiecchio A, Bozzo F, Motta M, Torchio B and Bocca C: COX-2 expression in human breast carcinomas: Correlation with clinicopathological features and prognostic molecular markers. Expert Opin Ther Targets 14: 655-664, 2010.
56. Harris RE, Casto BC and Harris ZM: Cyclooxygenase-2 and the inflammogenesis of breast cancer. World J Clin Oncol 5: 677-692, 2014.

57. DiDonato JA, Mercurio F and Karin M: NF- $\kappa \mathrm{B}$ and the link between inflammation and cancer. Immunol Rev 246: 379-400, 2012.

58. Liu J and Ma D: The Role of n-3 polyunsaturated fatty acids in the prevention and treatment of breast cancer. Nutrients 6 : 5184-5223, 2014

59. Culp BR, Titus BG and Lands WE: Inhibition of prostaglandin biosynthesis by eicosapentaenoic acid. Prostaglandins Med 3: 269-278, 1979 .

60. Hamid R, Singh J, Reddy BS and Cohen LA: Inhibition by dietary menhaden oil of cyclooxygenase- 1 and -2 in $\mathrm{N}$-nitrosomethylurea-induced rat mammary tumors. Int J Oncol 14: 523-528, 1999.

61. Ringbom T, Huss U, Stenholm Å, Flock S, Skattebøl L, Perera P and Bohlin L: COX-2 inhibitory effects of naturally occurring and modified fatty acids. J Nat Prod 64: 745-749, 2001

62. Patterson E, Wall R, Fitzgerald GF, Ross RP and Stanton C Health implications of high dietary omega- 6 polyunsaturated fatty acids. J Nutr Metab 2012: 539426, 2012.

63. Calder PC: n-3 fatty acids, inflammation and immunity: New mechanisms to explain old actions. Proc Nutr Soc 72: 326-336, 2013.

64. Yee LD, Agarwal D, Rosol TJ, Lehman A, Tian M, Hatton J, Heestand J, Belury MA and Clinton SK: The inhibition of early stages of HER-2/neu-mediated mammary carcinogenesis by dietary n-3 PUFAs. Mol Nutr Food Res 57: 320-327, 2013.

65. Liang M, Zhang P and Fu J: Up-regulation of LOX-1 expression by TNF-alpha promotes trans-endothelial migration of MDA-MB-231 breast cancer cells. Cancer Lett 258: 31-37, 2007.

66. Tavakoli-Yaraki M, Karami-Tehrani F, Salimi V and Sirati-Sabet M: Induction of apoptosis by Trichostatin A in human breast cancer cell lines: Involvement of 15-Lox-1. Tumor Biol 34: 241-249, 2013.

67. Moumen M, Chiche A, Decraene C, Petit V, Gandarillas A, Deugnier MA, Glukhova MA and Faraldo MM: Myc is required for $\beta$-catenin-mediated mammary stem cell amplification and tumorigenesis. Mol Cancer 12: 132, 2013.

68. Wen ZH, Su YC, Lai PL, Zhang Y, Xu YF, Zhao A, Yao GY, Jia CH, Lin J, Xu S, et al: Critical role of arachidonic acid-activated mTOR signaling in breast carcinogenesis and angiogenesis. Oncogene 32: 160-170, 2013

69. Sun H, Hu Y, Gu Z, Owens RT, Chen YQ and Edwards IJ: Omega-3 fatty acids induce apoptosis in human breast cancer cells and mouse mammary tissue through syndecan-1 inhibition of the MEK-Erk pathway. Carcinogenesis 32: 1518-1524, 2011.

70. Cao W, Ma Z, Rasenick MM, Yeh S and Yu J: N-3 poly-unsaturated fatty acids shift estrogen signaling to inhibit human breast cancer cell growth. PLoS One 7: e 52838, 2012.

71. Schley PD, Brindley DN and Field CJ: (n-3) PUFA alter raft lipid composition and decrease epidermal growth factor receptor levels in lipid rafts of human breast cancer cells. J Nutr 137: 548-553, 2007.

72. Bhargava R, Gerald WL, Li AR, Pan Q, Lal P, Ladanyi M and Chen B: EGFR gene amplification in breast cancer: Correlation with epidermal growth factor receptor mRNA and protein expression and HER-2 status and absence of EGFR-activating mutations. Mod Pathol 18: 1027-1033, 2005.

73. Zou Z, Bellenger S, Massey KA, Nicolaou A, Geissler A, Bidu C, Bonnotte B, Pierre AS, Minville-Walz M, Rialland M, et al: Inhibition of the HER2 pathway by $n-3$ polyunsaturated fatty acids prevents breast cancer in fat- 1 transgenic mice. J Lipid Res 54: 3453-3463, 2013.

74. Menéndez JA, Vázquez-Martín A, Ropero S, Colomer R and Lupu R: HER2 (erbB-2)-targeted effects of the omega-3 polyunsaturated fatty acid, alpha-linolenic acid (ALA; 18:3n-3) in breast cancer cells: The 'fat features' of the 'Mediterranean diet' as an 'anti-HER2 cocktail'. Clin Transl Oncol 8: 812-820, 2006.

75. Clay CE, Namen AM, Atsumi G, Willingham MC, High KP, Kute TE, Trimboli AJ, Fonteh AN, Dawson PA and Chilton FH: Influence of $\mathrm{J}$ series prostaglandins on apoptosis and tumorigenesis of breast cancer cells. Carcinogenesis 20: 1905-1911, 1999.

76. Tyagi S, Gupta P, Saini AS, Kaushal C and Sharma S: The peroxisome proliferator-activated receptor: A family of nuclear receptors role in various diseases. J Adv Pharm Technol Res 2: 236-240, 2011 
77. Rovito D, Giordano C, Vizza D, Plastina P, Barone I, Casaburi I, Lanzino M, De Amicis F, Sisci D, Mauro L, et al: Omega-3 PUFA ethanolamides DHEA and EPEA induce autophagy through PPAR $\gamma$ activation in MCF-7 breast cancer cells. J Cell Physiol 228: 1314-1322, 2013.

78. Bocca C, Bozzo F, Martinasso G, Canuto RA and Miglietta A Involvement of PPARalpha in the growth inhibitory effect of arachidonic acid on breast cancer cells. Br J Nutr 100: 739-750, 2008.

79. Chiu LC, Wong EY and Ooi VE: Docosahexaenoic acid from a cultured microalga inhibits cell growth and induces apoptosis by upregulating $\mathrm{Bax} / \mathrm{Bcl}-2$ ratio in human breast carcinoma MCF-7 cells. Ann N Y Acad Sci 1030: 361-368, 2004.

80. Jiang W, Zhu Z, McGinley JN, El Bayoumy K, Manni A and Thompson HJ: Identification of a molecular signature underlying inhibition of mammary carcinoma growth by dietary N-3 fatty acids. Cancer Res 72: 3795-3806, 2012.

81. Dozio E, Ruscica M, Passafaro L, Dogliotti G, Steffani L, Marthyn P, Pagani A, Demartini G, Esposti D, Fraschini F and Magni P: The natural antioxidant alpha-lipoic acid induces p27(Kip1)-dependent cell cycle arrest and apoptosis in MCF-7 human breast cancer cells. Eur J Pharmacol 641: 29-34, 2010.

82. Villegas-Comonfort S, Castillo-Sanchez R, Serna-Marquez N, Cortes-Reynosa P and Salazar EP: Arachidonic acid promotes migration and invasion through a PI3K/Akt-dependent pathway in MDA-MB-231 breast cancer cells. Prostaglandins Leukot Essent Fatty Acids 90: 169-177, 2014

83. Ravacci GR, Brentani MM, Tortelli T Jr, Torrinhas RS, Saldanha T, Torres EA and Waitzberg DL: Lipid raft disruption by docosahexaenoic acid induces apoptosis in transformed human mammary luminal epithelial cells harboring HER-2 overexpression. J Nutr Biochem 24: 505-515, 2013.

84. Horia E and Watkins BA: Complementary actions of docosahexaenoic acid and genistein on COX-2, PGE2 and invasiveness in MDA-MB-231 breast cancer cells. Carcinogenesis 28: 809-815, 2007.

85. Espinosa-Neira R, Mejia-Rangel J, Cortes-Reynosa P and Salazar EP: Linoleic acid induces an EMT-like process in mammary epithelial cells MCF10A. Int J Biochem Cell Biol 43 1782-1791, 2011.

86. Yerushalmi R, Woods R, Ravdin PM, Hayes MM and Gelmon KA: Ki67 in breast cancer: Prognostic and predictive potential. Lancet Oncol 11: 174-183, 2010.

87. Saggar JK, Chen J, Corey P and Thompson LU: Dietary flaxseed lignan or oil combined with tamoxifen treatment affects MCF-7 tumor growth through estrogen receptor- and growth factor-signaling pathways. Mol Nutr Food Res 54: 415-425, 2010

88. Truan JS, Chen JM and Thompson LU: Flaxseed oil reduces the growth of human breast tumors (MCF-7) at high levels of circulating estrogen. Mol Nutr Food Res 54: 1414-1421, 2010.

89. Taftachi R, Ayhan A, Ekici S, Ergen A and Ozen H Proliferating-cell nuclear antigen (PCNA) as an independent prognostic marker in patients after prostatectomy: A comparison of PCNA and Ki-67. BJU Int 95: 650-654, 2005.

90. Olivo SE and Hilakivi-Clarke L: Opposing effects of prepubertal low- and high-fat n-3 polyunsaturated fatty acid diets on rat mammary tumorigenesis. Carcinogenesis 26: 1563-1572, 2005.

91. Zhang P and Kong J: Doxorubicin-tethered fluorescent silica nanoparticles for $\mathrm{pH}$-responsive anticancer drug delivery. Talanta 134: 501-507, 2015.

92. Wiggins AK, Mason JK and Thompson LU: Growth and gene expression differ over time in alpha-linolenic acid treated breast cancer cells. Exp Cell Res 333: 147-154, 2015

93. Wu S, Guo Y, Wu Y, Zhu S, He Z and Chen YQ: Omega-3 free fatty acids inhibit tamoxifen-induced cell apoptosis. Biochem Biophys Res Commun 459: 294-299, 2015.

94. Chung H, Lee YS, Mayoral R, Oh DY, Siu JT, Webster NJ, Sears DD, Olefsky JM and Ellies LG: Omega-3 fatty acids reduce obesity-induced tumor progression independent of GPR120 in a mouse model of postmenopausal breast cancer Oncogene 34: 3504-3513, 2015.

95. Rose DP and Connolly JM: Effects of dietary omega-3 fatty acids on human breast cancer growth and metastases in nude mice. J Natl Cancer Inst 85: 1743-1747, 1993.

96. Rose DP, Connolly JM and Liu XH: Effects of linoleic acid and gamma-linolenic acid on the growth and metastasis of a human breast cancer cell line in nude mice and on its growth and invasive capacity in vitro. Nutr Cancer 24: 33-45, 1995.
97. Jiang WG, Hiscox S, Bryce RP, Horrobin DF and Mansel RE: The effects of n-6 polyunsaturated fatty acids on the expression of nm-23 in human cancer cells. Br J Cancer 77: 731-738, 1998.

98. Senzaki H, Iwamoto S, Ogura E, Kiyozuka Y, Arita S, Kurebayashi J, Takada H, Hioki K and Tsubura A: Dietary effects of fatty acids on growth and metastasis of KPL-1 human breast cancer cells in vivo and in vitro. Anticancer Res 18 $1621-1627,1998$

99. Mandal CC, Ghosh-Choudhury T, Yoneda T, Choudhury GG and Ghosh-Choudhury N: Fish oil prevents breast cancer cell metastasis to bone. Biochem Biophys Res Commun 402: 602-607, 2010

100. Kinoshita K, Noguchi M and Tanaka M: Effects of linoleic acid, eicosapentaenoic acid, and docosahexaenoic acid on the growth and metastasis of MM48 mammary tumor transplants in mice. Int J Oncol 8: 575-581, 1996

101. Kimura Y and Sumiyoshi M: Antitumor and antimetastatic actions of eicosapentaenoic acid ethylester and its by-products formed during accelerated stability testing. Cancer Sci 96: 441-450, 2005 .

102. Patterson RE, Flatt SW, Newman VA, Natarajan L, Rock CL, Thomson CA, Caan BJ, Parker BA and Pierce JP: Marine fatty acid intake is associated with breast cancer prognosis. J Nutr 141: 201-206, 2011.

103. Mareel M and Leroy A: Clinical, cellular, and molecular aspects of cancer invasion. Physiol Rev 83: 337-376, 2003.

104. Berindan-Neagoe I and Calin GA: Molecular pathways: microRNAs, cancer cells, and microenvironment. Clin Cancer Res 20: 6247-6253, 2014

105. Berindan-Neagoe I, Monroig Pdel C, Pasculli B and Calin GA MicroRNAome genome: A treasure for cancer diagnosis and therapy. CA Cancer J Clin 64: 311-336, 2014.

106. Braicu C, Tomuleasa C, Monroig P, Cucuianu A, Berindan-Neagoe I and Calin GA: Exosomes as divine messengers: Are they the Hermes of modern molecular oncology? Cell Death Differ 22: 34-45, 2015.

107. Fluckiger A, Dumont A, Derangère V, Rébé C, de Rosny C, Causse S, Thomas C, Apetoh L, Hichami A, Ghiringhelli F and Rialland M: Inhibition of colon cancer growth by docosahexaenoic acid involves autocrine production of TNFa. Oncogene 35: 4611-4622, 2016

108. Tang Y, Zhou X, Ji J, Chen L, Cao J, Luo J and Zhang S: High expression levels of miR-21 and miR-210 predict unfavorable survival in breast cancer: A systemic review and meta-analysis. Int J Biol Markers 30: e347-e358, 2015.

109. Hannafon BN, Carpenter KJ, Berry WL, Janknecht R, Dooley WC and Ding WQ: Exosome-mediated microRNA signaling from breast cancer cells is altered by the anti-angiogenesis agent docosahexaenoic acid (DHA). Mol Cancer 14: 133, 2015.

110. Mandal CC, Ghosh-Choudhury T, Dey N, Choudhury GG and Ghosh-Choudhury N: miR-21 is targeted by omega-3 polyunsaturated fatty acid to regulate breast tumor CSF-1 expression. Carcinogenesis 33: 1897-1908, 2012.

111. Shah MS, Davidson LA and Chapkin RS: Mechanistic insights into the role of microRNAs in cancer: Influence of nutrient crosstalk. Front Genet 3: 305, 2012.

112. Parasramka MA, Ho E, Williams DE and Dashwood RH MicroRNAs, diet and cancer: New mechanistic insights on the epigenetic actions of phytochemicals. Mol Carcinog 51: 213-230, 2012

113. Ortega FJ, Cardona-Alvarado MI, Mercader JM, Moreno-Navarrete JM, Moreno M, Sabater M, Fuentes-Batllevell N, Ramírez-Chávez E, Ricart W, Molina-Torres J, et al: Circulating profiling reveals the effect of a polyunsaturated fatty acid-enriched diet on common microRNAs. J Nutr Biochem 26: 1095-1101, 2015.

114. Farago N, Fehér LZ, Kitajka K, Das UN and Puskás LG: MicroRNA profile of polyunsaturated fatty acid treated glioma cells reveal apoptosis-specific expression changes. Lipids Health Dis 10: 173, 2011.

115. Chartoumpekis DV, Zaravinos A, Ziros PG, Iskrenova RP, Psyrogiannis AI, Kyriazopoulou VE and Habeos IG: Differential expression of microRNAs in adipose tissue after long-term high-fat diet-induced obesity in mice. PLoS One 7: e34872, 2012.

116. Davidson LA, Wang N, Shah MS, Lupton JR, Ivanov I and Chapkin RS: n-3 Polyunsaturated fatty acids modulate carcinogen-directed non-coding microRNA signatures in rat colon. Carcinogenesis 30: 2077-2084, 2009. 
117. Gil-Zamorano J,Martin R,Daimiel L, Richardson K, GiordanoE, Nicod N, García-Carrasco B, Soares SM, Iglesias-Gutiérrez E, Lasunción MA, et al: Docosahexaenoic acid modulates the enterocyte Caco-2 cell expression of microRNAs involved in lipid metabolism. J Nutr 144: 575-585, 2014.

118. Shukla K, Sharma AK, Ward A, Will R, Hielscher T, Balwierz A Breunig C, Münstermann E, König R, Keklikoglou I and Wiemann S: MicroRNA-30c-2-3p negatively regulates NF- $\kappa$ B signaling and cell cycle progression through downregulation of TRADD and CCNE1 in breast cancer. Mol Oncol 9: 1106-1119, 2015

119. Hu F, Meng X, Tong Q, Liang L, Xiang R, Zhu T and Yang S: BMP-6 inhibits cell proliferation by targeting microRNA-192 in breast cancer. Biochim Biophys Acta 1832: 2379-2390, 2013.

120. Baselga-Escudero L, Arola-Arnal A, Pascual-Serrano A, Ribas-Latre A, Casanova E, Salvadó MJ, Arola L and Blade C: Chronic administration of proanthocyanidins or docosahexaenoic acid reverses the increase of miR-33a and miR-122 in dyslipidemic obese rats. PLoS One 8: e69817, 2013.
121. Krishnamoorthy S, Recchiuti A, Chiang N, Fredman G and Serhan CN: Resolvin D1 receptor stereoselectivity and regulation of inflammation and proresolving microRNAs. Am J Pathol 180: 2018-2027, 2012.

122. Hardman WE, Sun L, Short N and Cameron IL: Dietary omega-3 fatty acids and ionizing irradiation on human breast cancer xenograft growth and angiogenesis. Cancer Cell Int 5: $12,2005$.

(i) (9) This work is licensed under a Creative Common International (CC BY-NC-ND 4.0) License. 